\title{
Seeing the Unseen: Autism Involves Reduced Susceptibility to Inattentional Blindness
}

\author{
John Swettenham ${ }^{1}$, Anna Remington ${ }^{2,3}$, Patrick Murphy ${ }^{1}$, Maike Feuerstein ${ }^{1}$, Kelly Grim ${ }^{1}$ and \\ Nilli Lavie ${ }^{2}$ \\ ${ }^{1}$ Developmental Science, University College London \\ ${ }^{2}$ Institute of Cognitive Neuroscience, University College London \\ ${ }^{3}$ Department of Experimental Psychology, University of Oxford \\ Word Count: 4,870
}

\section{Acknowledgements}

The authors would like to thank Dr Eleni Matechou for her advice regarding statistical analyses. Preparation of this article was supported by the Department of Developmental Science (JS) and the Scott Family Junior Research Fellowship (AR). We gratefully acknowledge the efforts of staff and pupils at schools in London who participated in the research, the National Autistic Society (UK) and the charity Resources for Autism.

Correspondence to: Dr John Swettenham, Developmental Science, University College London, Chandler House, 2 Wakefield Street, London WC1N 1PF. Phone: 0207679 4220. Email: j.swettenham@ucl.ac.uk 


\begin{abstract}
Objective: Attention research in individuals with autism spectrum disorder (ASD) has produced conflicting results. Some findings demonstrate greater distractibility whilst others suggest superior focused attention. Applying Lavie's load theory of attention to account for this discrepancy led us to hypothesize increased perceptual capacity in ASD. Preliminary support for our hypothesis has so far been found for adults with ASD with RT and signal detection sensitivity measures. Here we test the novel prediction we derived from this hypothesis that children with ASD should have lower rates of inattentional blindness than controls.
\end{abstract}

Method: 24 children with ASD (mean age 10 years, 10 months) and 39 typically-developing children (CA and NVMA matched) took part in the study. We assessed the effects of perceptual load on the rates of inattentional blindness in each group. Participants performing a line discrimination task in either a high load or low load condition were presented with an unexpected extra stimulus on a critical trial. Performance on the line judgement task and rates of detection and stimulus identification were recorded.

Results: Overall rates of detection and identification were higher in the ASD group than in the controls. Moreover, whereas both detection and identification rates were significantly lower in the high (compared to low) load conditions for the controls, these were unaffected by load in the ASD group.

Conclusion: Reduced inattentional blindness rates under load in ASD suggests higher perceptual capacity is a core feature, present from childhood and leading to superior performance in various measures of perception and attention.

Keywords: Autism Spectrum Disorder; Inattentional Blindness; Attention; Perceptual Load; Development 


\section{Introduction}

Research examining visual selective attention in individuals with autism spectrum disorder (ASD) has produced conflicting results. One set of findings demonstrates increased levels of distractibility in ASD relative to mental age matched controls (Burack, 1994; Christ, Holt, White, \& Green, 2007; Ciesielski, Courchesne, \& Elmasian, 1990), while other findings demonstrate superior performance on visual search tasks. For example, individuals with ASD are faster and more accurate than mental-age matched controls at locating an embedded figure within a line drawing (e.g. Shah \& Frith, 1993; Jolliffe \& Baron-Cohen, 1997), searching for local elements in a global figure (Plaisted, Swettenham, \& Rees, 1999) and finding a target presented among multiple non-target items (O'Riordan, Plaisted, Driver, \& Baron-Cohen, 2001; O'Riordan, 2004; Plaisted, O'Riordan, \& Baron-Cohen, 1998; Joseph, Keehn, Connolly, Wolfe, \& Horowitz, 2009). So, while a number of studies suggest visual selective attention is impaired in ASD leading to greater irrelevant distractor processing, performance on visual search tasks suggests superior visual selective attention.

Here we propose an account for this apparent discrepancy by applying Lavie's Load Theory of attention and cognitive control (e.g. Lavie, 2005) to ASD. According to Load Theory the extent of distractor processing depends on the level of perceptual load involved in the task. Perceptual load refers to the amount of task-relevant information, for example the number of different task stimuli or the perceptual requirements of a task (Lavie, 1995; Lavie \& Tsal, 1994). A high load task might consist of a large number of task-relevant items in a visual search array, or a subtle perceptual discrimination. A lower load task would consist of fewer items in a visual search array, or a more gross perceptual discrimination (e.g. Lavie, 2005; 2010). Central to the theory are the assumptions that while perception is a limited-capacity process, the processing of 


\section{Reduced Inattentional Blindness In Autism 4}

information that can be accommodated within perceptual capacity is mandatory. Consequently, perceptual processing proceeds on all items relevant to a current goal as well as those that are irrelevant until capacity is exhausted. Thus, in tasks of low perceptual load (e.g. requiring participants to identify a "target" stimulus from just two possible items) any spare capacity left over from the processing of task-relevant stimuli will 'spill over' into the processing of taskirrelevant distractor stimuli. In contrast, tasks involving high perceptual load (e.g. requiring search for a target stimulus in an array of four or more similar items) engage full perceptual capacity, leaving little or no room for distractor processing.

We reasoned that the pattern of reduced resistance to distractors on some selective visual attention tasks and enhanced performance on the visual search tasks may reflect a higher perceptual capacity in individuals with ASD compared to controls. Since Load Theory asserts that it is not possible to assign any less than total capacity, individuals with a higher perceptual capacity will necessarily process more information, whether relevant or irrelevant to the task. This accounts for superior performance on tasks where that further information is relevant to the search (Plaisted et al., 1998; O'Riordan et al., 2001; O'Riordan, 2004; Joseph et al., 2009), and increased distractor processing on tasks where the further information consists of irrelevant distractors (e.g. Remington, Swettenham, Campbell, \& Coleman, 2009; Burack, 1994).

We have provided preliminary evidence for this hypothesis by showing that increased levels of perceptual load in a search task (manipulated through the search set size) have led to reduced response competition effects produced by an irrelevant distractor in typical adults but not in adults with ASD. The latter continued to show a response competition effect at higher load, indicating that they perceived the distractor (Remington et al., 2009). This initial study, however, only provided indirect evidence of enhanced perceptual capacity in ASD as it relied on 
measuring an interference effect on reaction times which could also be influenced by various other factors such as effects on response selection rather than perception, and overall susceptibility to distraction. More direct support has been recently provided in a study that used a signal detection method to assess the effects of load on perceptual sensitivity (Remington, Swettenham, \& Lavie, 2012). The findings showed that increased levels of load in a visual search task led to reduced perceptual sensitivity (as measured with d') for typical adults, but left perceptual sensitivity unaffected in adults with ASD, in line with our load hypothesis.

Our previous findings may have important implications for the phenomenon of inattentional blindness (when people fail to notice a conspicuous visual event, for example a man dressed in a gorilla suit) in individuals with ASD. Since the level of perceptual load has been shown to be a critical factor in inattentional blindness (Cartwright-Finch \& Lavie, 2007), our hypothesis of enhanced capacity leads to the prediction that individuals with ASD would be less likely than matched controls to experience inattentional blindness in tasks of higher load. That is, they would show enhanced levels of awareness, being able to notice additional information when a task is taxing on capacity. The inattentional blindness paradigm is a well-established measure of awareness in the mainstream cognitive literature of attention. In order to assess awareness for a stimulus that should be entirely unattended participants are asked to attend to a primary task (e.g. discriminating line length) and an unexpected stimulus is then presented on the last trial in a task-irrelevant location (e.g. in the periphery for a task concerning the display centre). Awareness is then measured for this putatively unattended stimulus on that trial, by asking participants if they noticed anything extra on the last task display. The inattentional blindness measure is only based on that single report for each participant because once participants have been asked about their awareness one cannot be sure that the stimulus will be entirely unattended 
on subsequent trials, hence the single critical-trial nature of the paradigm.

In the present study we aimed to establish further our load hypothesis, testing the implications for perceptual awareness in children with ASD. Specifically we asked whether higher perceptual capacity is present already in childhood by assessing the effects of perceptual load on the extent to which children with ASD (compared to mental age matched controls) suffer from inattentional blindness or are able to notice an extra item that is not part of the task.

\section{Methods}

\section{Participants}

39 typically developing children and 28 children with a diagnosis of Autism Spectrum Disorder (ASD) participated in the study. The typically developing children attended an ordinary state local primary school. The children with ASD attended a specialist autism primary school that admits only children who have received a diagnosis of ASD. Informed consent was received in compliance with APA ethical guidelines. All participants with ASD had received a clinical diagnosis of ASD from a trained, independent clinician using the ADOS and following criteria listed in the Diagnostic and Statistical Manual of Mental Disorders, Fourth Edition (American Psychiatric Association, 1994). The diagnostic notes for the children with ASD indicated no other neurological or co-morbid psychiatric condition. The study used a between-participants design and participants within both groups were randomly allocated to either a high load or a low load condition. Participants were excluded if they obtained a score of less than five correct on the six non-critical trials, were incorrect on the critical trial, or were incorrect on a final control trial. These rather stringent exclusion criteria were used to ensure that all participants are engaging in 
the central task when the unexpected stimulus appears. Extensive piloting was conducted before task administration in the schools to increase the likelihood that most children would be able to perform the primary line-length discrimination task and so pass our stringent performance criterion. Overall 4 participants (all with ASD) scored less than five correct non-critical trials (3 from the low load condition and 1 from the high load condition) and therefore were removed from the sample. There may be a number of reasons why these 4 children performed poorly on the line discrimination task. For example they may have lacked motivation to perform the task or misunderstood the instructions. For the purposes of our experiment though, we only wished to include participants who had clearly engaged in the line-length discrimination (of either high or low load) at the same time that the irrelevant stimulus appeared.

The remaining participants were correct on the critical trial and were able to correctly detect and identify the critical stimulus in a final control trial, leading to no further exclusions. From the remaining 24 children with ASD, 11 were in the low load condition and 13 in the high load. From the 39 typically developing children 19 were in the low load condition and 20 in the high load condition. The ASD group was matched with the TD group for chronological age and for non-verbal IQ using the Raven's Standard Progressive Matrices (Raven, Raven, \& Court, 1998) (see table 1.) 
Table 1 Descriptive Statistics for each Group

\begin{tabular}{lllll}
\hline Group & Statistic & $\begin{array}{l}\text { Age } \\
\text { (years:months) }\end{array}$ & $\begin{array}{l}\text { Raven's } \\
\text { IQ }\end{array}$ & $\begin{array}{l}\text { Cross Judgement } \\
\text { Score (out of 7) }\end{array}$ \\
\hline ASD $(\mathrm{n}=24)$ & & & \\
Low Load $(\mathrm{n}=11)$ & Mean & $10: 09$ & 105.09 & 6.73 \\
& S.D. & $1: 5$ & 11.57 & 0.46 \\
& Range & $9: 2-13: 5$ & $90-135$ & \\
& & & & \\
High Load $(\mathrm{n}=13)$ & Mean & $10: 11$ & 104.78 & 6.15 \\
& S.D & $1: 6$ & 8.81 & 0.80 \\
& Range & $9: 9-13: 10$ & $90-110$ & \\
& & & & \\
\hline Control $(\mathrm{n}=39)$ & & & & \\
& & & 104.32 & 6.37 \\
Low Load $(\mathrm{n}=19)$ & Mean & $10: 10$ & 12.46 & 0.59 \\
& S.D. & $0: 8$ & $90-128$ & \\
& Range & $9: 10-11: 11$ & & \\
& & & 109.16 & 5.95 \\
High Load $(\mathrm{n}=20)$ & Mean & $10: 8$ & 11.48 & 0.69 \\
& S.D. & $0: 8$ & $92-126$ & \\
\hline
\end{tabular}

Independent samples t-tests indicated no significant difference between the groups overall or between subgroups in the low and high load conditions in Chronological age or Raven's IQ score, (all $\mathrm{p}$ values $>0.6)$. 
Stimuli and Procedure

Microsoft Visual Basic (version 6) was used to create computer-based stimuli that were presented on a 15 in. laptop screen (screen resolution 1024 x 768 pixels). Viewing distance was $60 \mathrm{~cm}$

In the high load condition, a target cross with a shorter arm subtending $3.35^{\circ}$ and a longer arm subtending $3.9^{\circ}$ appeared. In the low load condition, a target cross with a shorter arm subtending $1.25^{\circ}$ and a longer arm subtending $3.9^{\circ}$ appeared. Each cross was black and appeared on a white background. The longer line was either horizontal (H) or vertical (V) on each trial (3 $\mathrm{H}$ and $3 \mathrm{~V}$ appeared randomly across the first 6 trials, and either $\mathrm{H}$ or $\mathrm{V}$ counterbalanced across participants on trial 7). (see Figure 1a)

Each child was tested individually in a room at their school. They were first shown an example of each cross, presented on an A4 sheet of paper. The experimenter checked that the child could correctly report which line was longest in each example, either the line 'going across' or the line 'going up and down'. They were then told that crosses would be shown very briefly on the computer screen and for each cross they would be asked to indicate which line of the cross was longer.

A fixation circle subtending $0.15^{\circ}$ appeared at the centre of the screen at the beginning of each trial $(1500 \mathrm{~ms})$, followed by a blank display $(96 \mathrm{~ms})$, then a centrally located target cross (110ms) immediately followed by a visual mask (a mesh pattern of black lines on a white background, 496ms). The sequence ended with a blank screen which remained until the child had made a response. Participants in both conditions were required to judge whether the longer line was vertical or horizontal. The response could either be verbal (e.g. 'the line going across') or the participant could point to one of the two illustrations of the cross placed alongside the 
computer. The experimenter then entered the responses on the computer. Each participant completed seven trials.

On the seventh, critical trial, a black outline square shape (sides subtending $0.3^{\circ}$ ) appeared in addition to the cross. This critical stimulus appeared in one of four peripheral locations, counterbalanced between participants, in one of the four quadrants of the screen as defined by the lines of the cross. Each one of these locations was equidistant from fixation (at $3.2^{\circ}$ eccentricity) and equidistant from the two closest lines of the cross (see figure 1b).

Following the line judgement response on this trial, participants were asked whether they had noticed anything else appearing on the screen that had not been present before. If the participant reported seeing something they were asked to indicate where on the screen the critical stimulus had appeared. Participants were then presented with a choice of 4 shapes (see figure 1c) and asked which one of the shapes they had seen. 
Figure 1. a) Illustration of stimuli used in the low load condition (either one of the two top crosses shown here appears on each trial in random order) and the high load condition (one of the two bottom crosses appears on each trial in random order);

b) Illustration of a high load critical trial, with the critical stimulus (small square) in the top right

quadrant; c) Four shapes presented to participants who reported seeing the detection stimulus.

a)

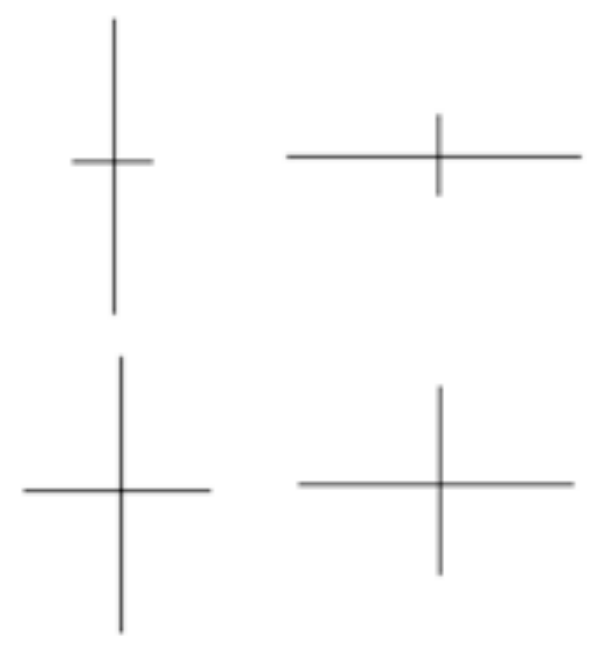

b)

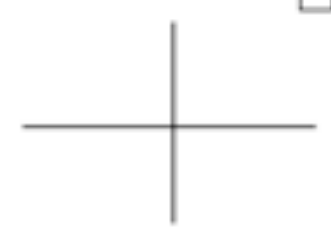

c)

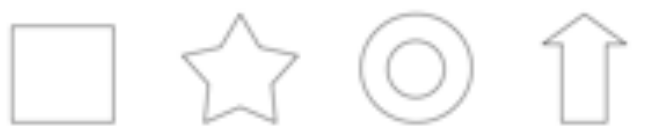


The critical trial was then repeated in a final control trial. Participants were told to ignore the cross and instead to look to see if they could see anything else on the screen. Reports of the quadrant where the critical stimulus appeared and the shape of the critical stimulus were again noted. Only participants who reported awareness of the critical stimulus and could identify location and shape of the critical stimulus were included in further analysis.

\section{Results}

\section{Task performance}

A two-way between-subjects ANOVA was conducted on the number of correct responses on the cross judgement task with the factors of diagnostic group (ASD vs. TD) and load condition (high vs. low). Mean and standard deviation detection and identification scores are shown in Table 1. There was a main effect of load $\left(\mathrm{F}(1,59)=8.50, \mathrm{p}=0.01, \eta_{\mathrm{p}}{ }^{2}=0.13\right)$ with participants in the low load condition scoring higher $(\mathrm{M}=6.50, \mathrm{SD}=0.57)$ than participants in the high load condition $(\mathrm{M}=6.03, \mathrm{SD}=0.73)$. There was no main effect of group $(\mathrm{F}(1,59)=$ $\left.2.74, \mathrm{p}=0.10, \eta_{\mathrm{p}}{ }^{2}=0.04\right)$ and no interaction between group and load $(\mathrm{F}(1,59)=0.208, \mathrm{p}=$ $\left.0.65, \eta_{\mathrm{p}}^{2}=0.004\right)$

These findings indicate that both groups engaged in task and that the manipulation of load was effective in increasing the task difficulty. (Recall that the numerically small effect of load on line-length discrimination is due to the restricted range enforced by our exclusion criteria, see the Method section). 
Awareness reports

Participants who reported that they had noticed something on the critical trial, and correctly identified in which quadrant the critical stimulus appeared, were classified as having detected the critical stimulus. Participants who were also able to identify the critical stimulus either by pointing to the correct shape from the four shapes presented, or by verbally describing a square, were classified as having correctly identified the critical stimulus. Figure 2 shows the percentage of participants from each group who were able to detect and identify the critical stimulus.

Figure 2. Percentage detection and identification rates for low and high load subgroups.

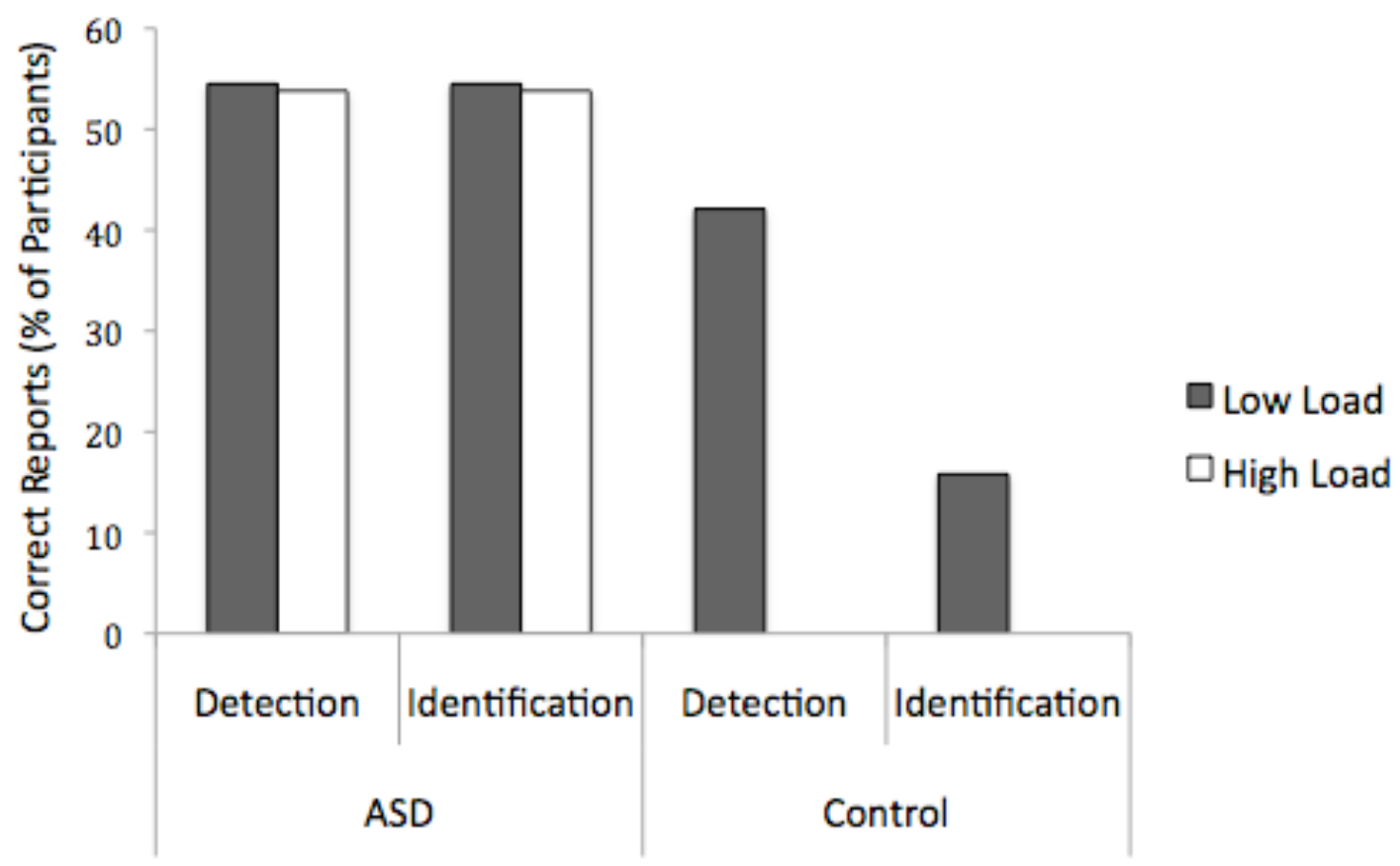

Detection.

Since this was a prospective binomial study design, a saturated logistic regression model was fitted 
(using IBM SPSS Statistics for Windows, Version 20.0) with the binary variable 'detection' as the response, and group and load as factors.

The likelihood ratio test indicated a significant interaction between group and load $\chi^{2}(1)=$ $7.95, p=.005$. This confirms a different effect of load on detection between the groups. Whereas for the controls detection rate in low load condition ( $42.1 \%$ of participants) was significantly higher than in high load ( $0 \%$ of participants) $\chi^{2}(1)=13.72, p<.001$; for the ASD group, there was no significant association between load and detection $\chi^{2}(1)=.001, p=.97(54.5 \%$ of participants in the low load condition, and $53.8 \%$ of participants in the high load condition) (see Fig 2).

In all conditions, detection rates were higher in the ASD group than control group resulting in overall detection rates of $54.2 \%$ and $20.5 \%$ respectively.

The significant reduction for the control group in rates of inattentional blindness from $42.1 \%$ in the low load conditions to 0 in the high load conditions confirms that our manipulation of task load was successful in determining rates of inattentional blindness in the TD population. The lack of effect of load on rates of detection in ASD (despite no greater deterioration in the linediscrimination task performance with higher load, as described in the previous section) is as predicted from our increased perceptual capacity hypothesis.

Identification.

The identification results showed a similar pattern to the detection results. The likelihood ratio test indicated an association between group and load that was approaching significance $\chi^{2}(1)=3.42$, $\mathrm{p}=.06$. Individual group analyses revealed a significant interaction between load and identification 
for the control group $\chi^{2}(1)=4.58, p=.03$, indicating a greater likelihood of correct identification in the low load (15.8\% of participants) than in the high load ( $0 \%$ of participants) conditions, and no association between load and identification for the ASD group (correct identification in 54.5\% of participants in the low load and $53.8 \%$ of participants in the high load conditions) $\chi^{2}(1)=.001, p=$ .973.

In all conditions, identification rates were higher in the ASD group than control group resulting in overall identification rates of $54.2 \%$ and $7.7 \%$ respectively.

\section{Discussion}

Our findings demonstrate that children with ASD have reduced rates of inattentional blindness and reduced impact of the level of perceptual load in a task on awareness, despite equally high levels of task performance as those of controls. These findings suggest that ASD involves increased visual perceptual capacity from childhood as we detail below.

The reduced rates of inattentional blindness found in ASD overall clearly show an advantage in perceptual awareness in the ASD group. Critically, while both groups showed a similarly high level of task performance in both high and low load conditions, the awareness rate was reduced by high load for typically developing children, whereas for the children with ASD the awareness rate was unaffected by the increased perceptual load of the primary visual task and remained high in the high load condition too.

Note that since the increased rate of awareness in ASD was not at the cost of performance on the primary line-length discrimination task itself, these findings suggest increased capacity. The children with ASD were able to detect the presence of an unexpected, task-irrelevant stimulus in addition to meaningfully engaging with the central task as shown by the finding of a 
main effect of load on the line discrimination performance for both groups. For the ASD group, the results were also equivalent across the measures of detection and identification judgments, in further support of enhanced perceptual capacity.

The current demonstration of reduced inattentional blindness under load in children with ASD is important because it suggests that higher perceptual capacity is already present in childhood, rather than emerging in adulthood as a result of atypical development. Previous demonstrations of increased perceptual capacity in ASD have been exclusively with adults (Remington et al., 2009; 2012). In addition, our group effect was found despite both groups being matched for performance on the Ravens Progressive Matrices (RPM). This strengthens our findings still further as ASD-related perceptual peaks are potentially accounted for in the RPM matching. If anything, one might expect a perceptual advantage to be masked by this matching.

Our increased perceptual capacity hypothesis not only accounts for the present findings but also may be able to account for the apparent discrepancy between previous reports of superior performance of individuals with ASD on visual attention (e.g. visual search and embedded figures), but reduced resistance to distractors on other selective attention tasks. According to load theory all stimuli in the visual field, regardless of their task relevance, will be processed automatically until perceptual capacity is exhausted. Applying load theory, we would therefore expect individuals with a higher perceptual capacity to process more information and therefore be less prone to inattentional blindness as we have shown. In cases where the information is task-relevant this would lead to superior performance, as is seen in visual search tasks and embedded figures tasks (Plaisted et al., 1998; O'Riordan et al., 2001; O'Riordan, 2004; Shah \& Frith, 1993; Shah \& Frith, 1983). Where the information is irrelevant, as in distractor tasks (Remington et al., 2009; Burack, 1994, Christ et al., 2007; Geurts et al., 2008; Adams \& 
Jarrold, 2012) then this would result in increased processing of distractor stimuli, leading some to propose that autism involves a distractability deficit. However, note that in our task the irrelevant stimulus was not designed to be distracting (e.g. was not response competing, cf. the flanker tasks). Our findings of an awareness advanatge in this task, therefore provides further support for our interpretation that both bodies of data demonstrate enhanced perceptual capacity rather than showing a reduced ability to control distraction.

Furthermore, our account leads to the specific prediction that differences between ASD and controls will be most apparent on visual search tasks when the perceptual load is high, allowing the ASD group to take advantage of their ability to process more information than controls. A close analysis of existing studies using the visual search paradigm shows that the data supports this prediction. For example, participants with ASD display superior performance when target-distractor similarity is high (O'Riordan \& Plaisted, 2001), when feature search is more difficult (O'Riordan et al., 2001; Kemner, van Ewijk, van Engeland, \& Hooge, 2008) and on conjunctive versus feature search tasks (Plaisted et al., 1998; O'Riordan, 2004). On all these tasks the ASD group advantage is also more prominent when array sizes are high and when the target is absent, conditions that would favour individuals with a high perceptual capacity as they involve the scanning of a large number of items.

For visual attention tasks that require participants to ignore irrelevant distractors our account also makes the specific prediction that group differences will be most apparent when perceptual load is high, this time with the ASD group showing higher levels of distraction than mental age matched controls. The logic here is that a high level of perceptual load in the central task will exhaust capacity in control participants, leaving no resources for distractor processing, but will not exhaust capacity in participants with ASD leaving spare capacity and allowing 
resources to spill over and automatically process irrelevant distractors. Again, existing studies confirm this prediction (Remington et al., 2009; Remington et al., 2012), and the present findings show a similar pattern.

Our account and present results might also explain why previous studies examining susceptibility to change blindness in ASD have produced equivocal results. Change blindness refers to the difficulty participants have in detecting the difference between two pictures when they are presented successively with a brief interruption (Simons \& Levin, 1997). Group differences have been reported when complex scenes (high load) are used (Smith \& Milne, 2009; Fletcher-Watson et al., 2012): Children with ASD detect the change more quickly than controls under such conditions. However, when pairs of images of isolated objects (low load) were used in a change blindness task, no group difference were found (Burack et al., 2009). Again, our increased perceptual capacity hypothesis of ASD would predict these findings, since the higher capacity in the ASD participants would lead to the processing of more stimuli in a high perceptual load scene leading to faster detection of the changed item. This advantage would be absent in a low perceptual load scene since only a limited number of items need to be processed and this can be achieved equally by both ASD children and controls.

The single trial nature of the inattentional blindness paradigm means that this study relies on a small data set. We should therefore be cautious not to overstate the importance of the findings from this study alone. We note however that the convergence of the present results with previous results in studies assessing both distractor processing and detection as a function of load in ASD, that have used longer versions of load tasks (Remington et al 2009; Remington, Swettenham \& Lavie, 2012), further adds support for our interpretation.

Currently there are no established measures that directly assess visual perceptual capacity 
in the mainstream attention and visual perception literature. In the absence of such measures researchers typically rely on measures such as failure or success in dual task performance similarly to our current study design (see also Macdonald \& Lavie, 2008 and Remington et al., 2012 in the case of ASD). In line with this rationale, the results from the present study as well as our previous studies are most likely to be explained by an enhanced perceptual capacity. In each case continued processing of additional stimuli that are not part of the primary task is found at higher levels of load in ASD compared to controls, whilst there are no group differences at low levels of load. Nevertheless, the goal of establishing direct measures of perceptual capacity is an important direction for future research

Unfortunately, an increased perceptual capacity in ASD is unlikely to have an impact on the ability to learn what is relevant and what is not, even though it may mean processing more information. In load theory, perceptual processing capacity and the ability to prioritise the focus of attention on relevant rather than irrelevant information are considered to be two distinct functions. The function of paying attention to what is relevant and what is irrelevant is considered a high-level cognitive control function that has been shown to be dissociable from perceptual capacity (e.g. Lavie, 2000; Lavie, Hirst, De Fockert \& Viding, 2004).

What neural mechanisms might underlie the increased perceptual capacity in ASD? One clue comes from research with typical adults on the neural basis of processing load. It is now well established that perceptual load in the context of a visual search task elicits responses in the posterior parietal cortex, especially the inferior parietal sulcus (IPS) (Wojciulik \& Kanwisher 1999). More recent studies have established a neural signature of gradual increases in perceptual load in the IPS, using tasks such as visual search or motion tracking (Mitchell and Cusak 2008; Jovovich et al 2001). Furthermore, this increase in parietal activity is accompanied by reduced 
baseline level of visual cortex excitability in areas unrelated to the task (Muggleton, Lamb, Ealsh, Walsh \& Lavie, 2008; Carmel, Thorne, Rees \& Lavie, 2011) and a reduction in visual cortex activity in response to task-irrelevant stimuli (Bahrami, Lavie \& Rees, 2007; Pinsk, Doniger \& Kastner, 2004; Schwartz et al, 2005; Yi, Woodman, Widders, Marois \& Chun, 2004). These findings suggest that neural activity in parietal and visual cortical areas mediates perceptual capacity in neurotypical individuals.

Given the likely neural mechanism in typical adults, we predict that in ASD increased perceptual capacity may be related to greater availability of parietal and visual cortex resources. Consitent with this proposal are anotomical observations of increased gray matter volume in the parietal cortex in ASD (Ashitari et al, 2007; Brieber et al, 2007) and evidence of overconnectivity in the visual cortex (Just, Cherkassky, Keller, Kana \& Minchew, 2007; Keita, Mottron \& Bertone, 2010). Furthermore, functional neuroimaging has revealed stronger activation in visual cortex on various perceptual tasks in which individuals with ASD display behavioural superiorities, such as visual search (Keehn et al, 2008) embedded figures (Manjaly et al, 2007; Ring et al, 1999), block design (Hubl et al, 2003) and matrix reasoning (Soulieres et al, 2009). It will be important to directly test this proposal in future studies by measuring cortical activity while manipulating load in ASD.

We have now demonstrated, through our behavioural studies, increased perceptual capacity in both adults and children with ASD. Support for our account with data from children suggests that increased capacity may be a core feature of the disorder rather than a result of unusual environmental input. For example, a well known feature of ASD is a preference for processing 'local' rather than 'global' information (Happe and Frith, 1990). This preference could be considered a phenomenon of increased perceptual capacity, alternatively the preference 
to attend to detail could contribute to the development of an increased perceptual capacity, being a cause rather than consequence. Future studies testing our account with even younger populations may be useful in furthering our understanding of the origins of increased perceptual capacity.

We also note that our studies with both adults and children with ASD have so far only involved intellectually able individuals (IQ > 90) and have not involved individuals with a dual diagnosis (e.g. ASD and attention deficit disorder). This might therefore be considered a select group. In addition, we have not yet included other developmentally disordered groups as a comparison. Future research may establish whether increased perceptual capacity is specific to ASD and whether only intellectually able individuals with ASD have increased perceptual capacity.

Finally, a greater understanding of what characterizes the unusual attentional and perceptual style may help in the design of educational and therapeutic programs which capitalize on the relative strengths as well as the weakness of ASD. 


\section{Reference List}

Adams, N. C. \& Jarrold, C. (2012). Inhibition in Autism: Children with Autism have Difficulty Inhibiting Irrelevant Distractors but not Prepotent Responses. Journal of Autism and Developmental Disorders. 42,1052 - 1063.

American Psychiatric Association (1994). DSM-IV Diagnostic and Statistical Manual of Mental Disorders. (4th ed.) Washington DC: American Psychiatric Association.

Ashtari, M., Nichols, S., McIlree, C., Spritzer, L., Adesman, A., \& Ardekani, B. (2007). Novel imaging technique shows gray matter increase in brains of autistic children. In Annual Meeting of the Radiological Society of North America.

Bahrami, B., Lavie, N., \& Rees, G. (2007). Attentional load modulates responses of human primary visual cortex to invisible stimuli. Current Biology, 17, 509-513.

Brieber, S., Neufang, S., Bruning, N., Kamp-Becker, I., Remschmidt, H., HerpertzDahlmann, B. et al. (2007). Structural brain abnormalities in adolescents with autism spectrum disorder and patients with attention deficit/hyperactivity disorder. Journal of Child Psychology and Psychiatry, 48, 1251-1258.

Burack, J., Joseph, S., Russo, N., Shore, D. I., Porporino, M., \& Enns, J. (2009). Change detection in naturalistic pictures among children with autism. Journal of Autism and Developmental Disorders, 39, 471-479.

Burack, J. A. (1994). Selective attention deficits in persons with autism: preliminary evidence of an inefficient attentional lens. Journal of Abnormal Psychology., 103, 535-543. 
Carmel, D., Thorne, J. D., Rees, G., \& Lavie, N. (2011). Perceptual load alters visual excitability. Journal of Experimental Psychology Human Perception and Performance, 37, $1350-1360$.

Cartwright-Finch, U. \& Lavie, N. (2007). The role of perceptual load in inattentional blindness. Cognition, 102, 321-340.

Christ, S. E., Holt, D. D., White, D. A., \& Green, L. (2007). Inhibitory control in children with autism spectrum disorder. Journal of Autism and Developmental Disorders, 37, 1165.

Ciesielski, K. T., Courchesne, E., \& Elmasian, R. (1990). Effects of focused selective attention tasks on event-related potentials in autistic and normal individuals. Electroencephalography and Clinical Neurophysiology, 75, 207-220.

Fletcher-Watson, S., Leekam, S. R., Connolly, B., Collis, J. M., Findlay, J. M., McConachie, H. et al. (2012). Attenuation of change blindness in children with autism spectrum disorders. British Journal of Developmental Psychology, 30, 446-458.

Happe, F. \& Frith, U. (2006). The weak central coherence account: detail-focused cognitive style in autism spectrum disorders. Journal of Autism and Developmental Disorders, $36,5-25$.

Hauck, W. W. \& Donner, A. (1977). Wald's test as applied to hypotheses in logit analysis. Journal of the American Statistical Association, 72, 851-853.

Hubl, D., Bolte, S., Feineis-Matthews, S., Lanfermann, H., Federspiel, A., Strik, W. et al. (2003). Functional imbalance of visual pathways indicates alternative face processing strategies in autism. Neurology, 61, 1232-1237. 
Jolliffe, T. \& Baron-Cohen, S. (1997). Are people with autism and Asperger syndrome faster than normal on the Embedded Figures Test? Journal of Child Psychology and Psychiatry, $38,527-534$.

Joseph, R., Keehn, B., Connolly, C., Wolfe, J., \& Horowitz, T. (2009). Why is visual search superior in autism spectrum disorder? Developmental Science, 12, 1083-1096.

Jovovich, J., Peters, R. J., Koch, C., Braun, J., Chang, L., \& Ernst, T. (2001). Brain areas specific for attentional load in a motion-tracking task. Journal of Cognitive Neuroscience, 13, 1048-1058.

Just, M. A., Cherkassky, V. L., Keller, T. A., Kana, R. K., \& Minshew, N. J. (2007). Functional and anatomical cortical underconnectivity in autism: evidence from an FMRI study of an executive function task and corpus callosum morphometry. Cerebral Cortex, 17, 951-961.

Keehn, B., Brenner, L., Palmer, E., Lincoln, A. J., \& Muller, R. A. (2008). Functional brain organization for visual search in ASD. Journal of the International Neuropsychological Society, 14, 990-1003.

Keita, L., Mottron, L., \& Bertone, A. (2010). Far visual acuity is unremarkable in autism: do we need to focus on crowding? Autism Research, 3, 333-341.

Kemner, C., van Ewijk, L., van Engeland, H., \& Hooge, I. (2008). Brief report: Eye movements during visual search tasks indicate enhanced stimulus discriminability in subjects with PDD. Journal of Autism and Developmental Disorders, 38, 553-557.

Lavie, N. (1995). Perceptual load as a necessary condition for selective attention. Journal of Experimental Psychology: Human Perception and Performance, 21, 451-468. 
Lavie, N. (2000). Selective attention and cognitive control: Dissociating attentional functions through different types of load. In Attention and performance XVIII (Monsell, S. and Driver, J., eds), pp. 175-194, MIT Press

Lavie, N. (2005). Distracted and confused?: selective attention under load. Trends in Cognitive Science, 9, 75-82.

Lavie (2010). Attention, Distraction, and Cognitive Control Under Load. Current Directions in Psychological Science, 19, 143-148.

Lavie, N., Hirst, A., de Fockert, J. W., \& Viding, E. (2004). Load theory of selective attention and cognitive control. Journal of Experimental Psychology: General, 133, 339-354.

Lavie, N., \& Tsal, Y. (1994). Perceptual load as a major determinant of the locus of selection in visual attention. Perception and Psychophysics, 56, 183-197.

Manjaly, Z. M., Bruning, N., Neufang, S., Stephan, K. E., Brieber, S., Marshall, J. C. et al. (2007). Neurophysiological correlates of relatively enhanced local visual search in autistic adolescents. Neuroimage., 35, 283-291.

Macdonald, J. S. \& Lavie, N. (2008). Load induced blindness. Journal of Experimental Psychology: Human Perception and Performance, 34, 1078-1091.

Mitchell, D. J. \& Cusack, R. (2008). Flexible, Capacity-Limited Activity of Posterior Parietal Cortex in Perceptual as well as Visual Short-Term Memory Tasks. Cerebral Cortex, 18, 1788-1798.

Muggleton, N., Lamb, R., Walsh, V., \& Lavie, N. (2008). Perceptual load modulates visual cortex excitability to magnetic stimulation. Journal of Neurophysiology, 100, 516-519.

O'Riordan, M. \& Plaisted, K. (2001). Enhanced discrimination in autism. Quaterly Journal of Experimental Psychology: A, 54, 961-979. 
O'Riordan, M. A. (2004). Superior visual search in adults with autism. Autism, 8, 229248.

O'Riordan, M. A., Plaisted, K. C., Driver, J., \& Baron-Cohen, S. (2001). Superior visual search in autism. Journal of Experimental Psychology Human Perception and Performance, 27, 719-730.

Pinsk, M. A., Doniger, G. M., \& Kastner, S. (2004). Push-pull mechanism of selective attention in human extrastriate cortex. Journal of Neurophysiology, 92, 622-629.

Plaisted, K., O'Riordan, M., \& Baron-Cohen, S. (1998). Enhanced visual search for a conjunctive target in autism: a research note. Journal of Child Psychology and Psychiatry, 39, $777-783$.

Plaisted, K., Swettenham, J., \& Rees, L. (1999). Children with autism show local precedence in a divided attention task and global precedence in a selective attention task. Journal of Child Psychology and Psychiatry, 40, 733-742.

R Development Core Team (2008). $R$ : A language and environment for statistical computing. R Foundation for Statistical Computing, Vienna, Austria.

Raven, J., Raven, J. C., \& Court, J. H. (1998). Manual for Raven'sprogressivematrices and vocabulary scales—section 1: general overview. Oxford: Oxford Psychologists Press.

Remington, A., Swettenham, J., Campbell, R., \& Coleman, M. (2009). Selective attention and perceptual load in autism spectrum disorder. Psychological Science, 20, 1388-1393.

Remington, A. M., Swettenham, J. G., \& Lavie, N. (2012). Lightening the load: perceptual load impairs visual detection in typical adults but not in autism. Journal of Abnormal Psychology, 121, 544-551. 
Ring, H., Baron-Cohen, S., Wheelwright, S., Williams, S. C., Brammer, M., Andrew, C. et al. (1999). Cerebral correlates of preserved cognitive skills in autism. A functional MRI study of Embedded Figures Task performance. Brain, 122, 1305-1315.

Schwartz, S., Vuilleumier, P., Hutton, C., Maravita, A., Dolan, R., \& Driver, J. (2005). Attentional load and sensory competition in human vision: Modulation of fMRI responses by load at fixation during task-irrelevant stimulation in the peripheral visual field. Cerebral Cortex, $15,770-786$.

Shah, A. \& Frith, U. (1983). An islet of ability in autistic children: a research note. Journal of Child Psychology and Psychiatry, 24, 613-620.

Shah, A. \& Frith, U. (1993). Why do autistic individuals show superior performance on the block design task? Journal of Child Psychology and Psychiatry, 34, 1351-1364.

Simons, D. J. \& Levin, D. T. (1997). Change blindness. Trends in Cognitive Science, 1, 261-267.

Smith, H. \& Milne, E. (2009). Reduced change blindness suggests enhanced attention to detail in individuals with autism. Journal of Child Psychology and Psychiatry, 50, 300-306.

Soulieres, I., Dawson, M., Samson, F., Barbeau, E. B., Sahyoun, C. P., Strangman, G. E. et al. (2009). Enhanced visual processing contributes to matrix reasoning in autism. Human Brain Mapping, 30, 4082-4107.

Venables, W. N. \& Ripley, B. D. (2003). Modern Applied Statistics with S. New York,US: Springer.

Wojciulik, E. \& Kanwisher, N. (1999). The generality of parietal involvement in visual attention. Neuron, 23, 747-764. 
Yi, D. J., Woodman, G. F., Widders, D., Marois, R., \& Chun, M. M. (2004). Neural fate of ignored stimuli: Dissociable effects of perceptual and working memory load. Nature Neuroscience, 7, 992-996. 